\title{
Spontaneous Absorption, Einstein's Rate Equation Approximation and Zero Point Energy
}

\author{
J. Saikia ${ }^{1^{*}}$ and G.D. Baruah ${ }^{2}$ \\ ${ }^{1}$ Department of Physics, J.B. College (Autonomous), Jorhat, Assam, India \\ ${ }^{2}$ Centre for Laser \& Optical Science, New Uchamati, Doom Dooma, Assam, India \\ *Corresponding author's E-mail: jibonsaikiajbc@gmail.com Mobile +919435351338
}

Available online at: www.isroset.org

Received: 10/Feb/2019, Accepted: 24/Feb/2019, Online: 28/Feb/2019

\begin{abstract}
Einstein's derivation of Planck's black body energy distribution law has been examined with the inclusion of the process of spontaneous absorption. It has been shown that the inclusion of the term of spontaneous absorption does not affect the distribution law but leads to an equation which was derived by Max Plank in 1911.The idea of relating spontaneous absorption to zero point energy is discussed in its historical prelude. We have observed that in the steady state temperature $\mathrm{T}$, inclusion of the term of spontaneous absorption with some arbitrarily chosen value of transition probability results in the same distribution law for the black body as derived by Einstein in 1917, plus an additional term which points to the universal, uniform, all-pervasive phenomenon of zero point energy. The physics of zero point energy is well known but the zero point energy that originates from the term of spontaneous absorption is completely new. It is reasonable to believe that spontaneous is non-zero for a ground state which is not perfectly vacuum.
\end{abstract}

Key Words: Spontaneous absorption, Spontaneous emission, Zero point energy, Transition probability

\section{INTRODUCTION}

Spontaneous radiation, together with stimulated radiation and absorption is among the most fundamental processes in quantum optics. These processes are primarily responsible for the interaction of radiation with matter. Light falling on matter is absorbed leaving the atoms in the excited state which spontaneously emits radiation with a spread of frequency inverse by proportional to the decay time. The situation is modified when the atomic decay times are sufficiently long and the radiation is sufficiently intense. The radiation falling on the excited state of the atom results in the stimulated emission rather than stimulated absorption. This process of stimulated emission is needed to obtain Planck's radiation law in Einstein derivation [1]. It is stimulated emission which enables an atomic medium to amplify incident radiation and therefore is an essential part in light amplifiers and oscillators. Quantum theory of radiation [2, 3] explains spontaneous emission as a consequence of induced zero-point oscillation of electromagnetic field of empty space. This mechanism of spontaneous emission- an isotropic perturbation, always present, attributed in connection with the quantum theory to the all pervading zero point fluctuations of the electromagnetic field. The light excites the atom-the zero point fluctuation de-excites them, resulting in the reradiation of light. According to Ginzburg [4], the definition is equally applicable in the classical and quantum regions. According to Heitler [3] the interaction between the atom and the radiation field can cause these radiative transitions even if, in the initial state, no light quanta at all are present.

The zero-point oscillations are a purely quantum mechanical effect which is not actually present in the classical theory and which formally disappears as $\hbar \rightarrow 0$. Zero point fluctuations give rise to the effect like Lamb shift [5], Casimir effect [6], Laser line width [7] and the spontaneous emission. Stimulated emission has been observed as LASER and is now available in many forms over a wide spectral range from for ultraviolet and X-ray to infrared. Similarly absorption (or stimulated absorption) is ubiquitous and it is easily observed as dark lines in the absorption spectra. In case of spontaneous absorption it is worthwhile to note that nobody has observed it and adequate discussion on this topic has not been found in any available literature. In the present work we shall specifically consider Einstein's rate equation where the phenomenon of spontaneous absorption is also included to work out the radiation formula. We shall see in section 2 that this leads to an equation which is derived by Planck [8] in 1911.

We organize the paper as follows. In section 2 the phenomena of spontaneous emission and spontaneous 
absorption are discussed as a historical prelude. It imitates the justification of our work. In section 3 Einstein's rate equation approximation has been used with the process of spontaneous absorption being included. Section 4 is a concluding discussion.

\section{II .SPONTANEOUS EMISSION AND SPONTANEOUS ABSORPTION}

We note here that although zero-point energy is usually regarded as a quantum phenomenon and a consequence of the Heisenberg uncertainty principle, the existence of zero-point energy was inferred by Plank, Einstein and others in the context of black body radiation prior to the discovery of quantum mechanics, Einstein and Otto Stern [9] came close to the derivation of black body radiation function without assuming quantization but with the presence of zero-point energy. Because of the dissatisfaction, Planck in 1910 formulated and worked out this second theory regarding black body radiation formula which indicates directly the truth of the existence of all pervading zero point energy. Planck's equation for the energy density $\rho$ of a blackbody is of the form.

$$
\rho(v, T)=\frac{g m v^{2}}{c^{3}}\left\{\frac{h w}{g^{h v} \| k T-1}+\frac{h v}{2}\right\} d v
$$

This equation has the same temperature depended term as derived in the first theory plus an additional term $\frac{1}{2} \mathrm{hv}$. In Equation (1) $v$ is the frequency, $\mathrm{c}$ is the speed of light and $\mathrm{k}$ is the Boltzmann constant. If the temperature $\mathrm{T}$ in Equation (1) drops to zero we are left with the zero point term.

Based on the work of Ginzburg [4] and Miloni [10] we find it justified to include the term of spontaneous absorption in Einstein's rate equation leading to the radiation law of Planck in 1917. In this connection it is worthwhile to consider the perennial question, why there is a need to relate spontaneous radiation to zero-point oscillation. This is indicated by Weisskopf [11] in a review in 1935. He took an active part in the development of quantum theory. In [11] Weisskopf writes, "From the quantum theory there follows the existence of so-called zero point oscillations, for example each oscillation in its lowest position is not completely at rest but always is moving about the equilibrium position. Therefore electromagnetic oscillations; for example each oscillation in its lowest position is not completely at rest but always is moving about the equilibrium position. Therefore electromagnetic oscillations also never cease completely. Thus the quantum nature of the electromagnetic field has as its consequence zero point oscillations of the field strength in the lowest energy state, in which there is no light quanta in space."

"The zero point oscillations act on an electron in the same way as ordinary electrical oscillations do. They can change the eigen state of the electron, but only in a transition to a state with lowest energy, since empty space can only take away energy, and not give it up. In this way spontaneous radiation arises as a consequence of the existence of this unique field strengths corresponding to the zero point oscillations. Thus spontaneous radiation is the induced radiation of light quanta produced by light quanta of empty space." Research on the lamb shift led to the idea that this shift of an energy level and also the natural line with due to spontaneous emission could be attributed to the quantum mechanical zero-point fluctuations of the electromagnetic field. Welton [12] wrote that spontaneous emission can be thought of as forced emission taking place under the action of the fluctuating field.

From what has been discussed above it is understand that the zero point oscillation is concerned only in the case of a transition to state with lowest energy i.e. spontaneous emission. But in the case of a space which is not perfectly empty this statement needs to be qualified. Fain et. al.(13) has shown that in the ground state the influence of vacuum field fluctuations is cancelled by the atomic dipole fluctuations. With these backgrounds we make a bold assumption that the probability of spontaneous absorption is non zero and proceed to derive Planck's radiation law using Einstein's rate equation approximation.

\section{SPONTANEOUS ABSORPTION AND EINSTEIN'S RATE EQUATION}

Before the creation of quantum mechanics in the frame work of old quantum theory the major problem was the description of the absorption and emission of light by an atom. In the absence of a good microscope theory the only approach was a detailed discussion involving emission and absorption probabilities. This was done by Einstein [1], who explicitly indicated an analogy with radioactive decay.

The experimentally observed spectral distribution of black body radiation is well fitted by the formula discovers by Planck

$$
\rho(v, \mathrm{~T}) \mathrm{d} v=\frac{\mathrm{B \pi} v^{2}}{c^{3}} \frac{k v}{\mathrm{~g}^{h w} \hat{k} \mathrm{kT}-1} \mathrm{~d} v
$$

where $v$ is frequency in Hertz, $\mathrm{T}$ is the absolute temperature, $\mathrm{k}$ is the Boltzmann and $\mathrm{c}$ is the speed of light. Einstein revealed that this formula can be explained in terms of ensemble of atoms with discrete energy levels of various frequencies provided that spontaneous emission is included along with the process of stimulated emission and stimulated absorption [2]. The upper and ground levels are populated according to the Boltzman distribution. In this section we follow this derivation but include information of spontaneous absorption. 
We consider appropriate expressions related to transitions in a system of atoms between states $\mathrm{n}$ and $\mathrm{m}$ with energies $E_{n}$ and $E_{m}\left(>E_{n}\right)$ and consider as in the work of Einstein the radiation to be isotropic, unpolarized and to be propagating in vacuum. Generalization to the case of presence of a medium and with the inclusion of various polarizations of the normal modes is given by Ginzburg [14]. Let us assume that there are $\mathrm{n}_{1}$ atoms in the lower state and $\mathrm{n}_{2}$ atoms in the upper state and $\rho(v)$ is the radiation field density we thus have.

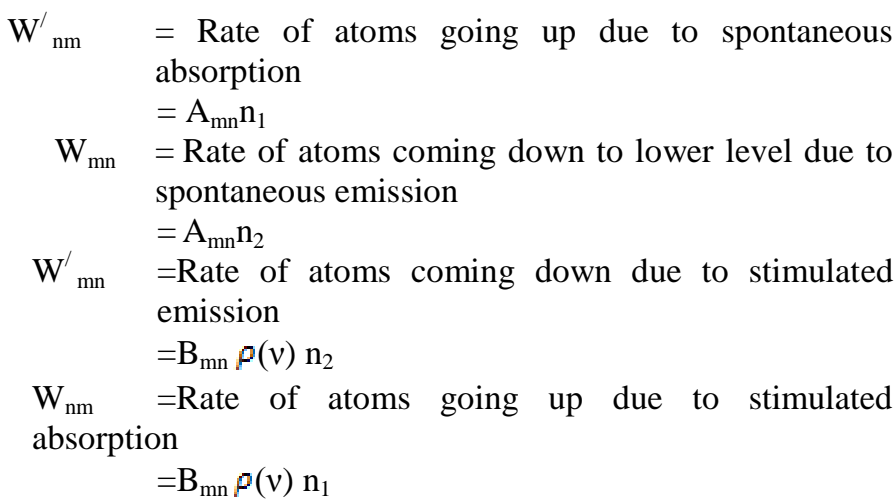

At this stage we would like to emphasize the fact that in a laser the spontaneous emission always appears as a noise and until now nobody has been able to separate this noise from stimulated emission. Similar considerations may be applicable in the case of stimulated absorption where spontaneous absorption appears as a noise.

The word stimulated absorption has been used in place of "absorption" simply because of the fact that when a radiation of photon density $\rho(v)$ of right frequency is incident on the lower level $\mathrm{n}_{1}$ the atoms in that level receive the radiation absorb it and get stimulated to the higher level $\mathrm{n}_{2}$. This is just the mirror image of stimulated emission. We thus have the basic assumptions.

$$
\begin{aligned}
& \mathrm{n}_{2}=\mathrm{n}_{1} \exp (-\mathrm{h} v / \mathrm{kT}) \\
& \mathrm{h} v=\mathrm{E}_{\mathrm{m}}-\mathrm{E}_{\mathrm{n}} \\
& \mathrm{W}_{\mathrm{nm}}+\mathrm{W}^{\prime}{ }_{\mathrm{nm}}=\mathrm{W}_{\mathrm{mn}}+\mathrm{W}^{\prime}{ }_{\mathrm{mn}}
\end{aligned}
$$

The second term in the LHS is new

$$
\begin{aligned}
& \mathrm{n}_{2} \mathrm{~A}_{\mathrm{mn}}+\mathrm{B}_{\mathrm{mn}} \rho(v) \mathrm{n}_{2}=\mathrm{n}_{1} \rho(v) \mathrm{B}_{\mathrm{nm}}+\mathrm{n}_{1} \mathrm{~A}_{\mathrm{nm}} \\
& \rho(v)\left\{\mathrm{n}_{1} \mathrm{~B}_{\mathrm{nm}}-\mathrm{n}_{2} \mathrm{~B}_{\mathrm{nm}}\right\}=\mathrm{n}_{2} \mathrm{~A}_{\mathrm{nm}}+\mathrm{n}_{1} \mathrm{~A}_{\mathrm{nm}} \\
& \rho(v)\left\{\frac{\mathrm{n}_{1}}{\mathrm{n}_{2}} B_{\mathrm{nm}}-B_{m n}\right]=A_{m n}+\frac{\mathrm{n}_{1}}{\mathrm{n}_{2}} A_{\mathrm{nm}} \\
& \rho(v)\left\{\exp (\mathrm{h} v \mid \mathrm{kT}) \mathrm{B}_{\mathrm{nm}}-\mathrm{B}_{\mathrm{mn}}\right\}=A_{m m}+ \\
& \exp (h v / k T) A_{n m}
\end{aligned}
$$

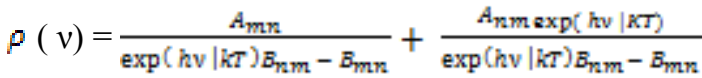

$$
\begin{aligned}
& =\frac{A}{B} \frac{1}{\exp (\hbar v \mid k T)-1}+\frac{A}{2 B} \frac{1}{1-\exp (-\hbar v \mid k T)} \\
& =\frac{A}{B}\left\{\frac{1}{\exp (h w \mid k T)-1}+\frac{1}{2} \frac{1}{1-\exp (-h w \mid k T)}\right\} \\
& \rho(v) \mathrm{d} v=\frac{\operatorname{B\pi h} v^{3}}{c^{3}}\left\{\frac{1}{\exp (\hbar k \mid k T)-1}+\frac{1}{2} \frac{1}{1-\exp (-\hat{h} v \mid k T)}\right\} \mathrm{d} v \\
& =\frac{8 \pi h v^{2}}{c^{3}}\left\{\frac{\hbar k v}{\exp (h w \mid k T)-1}+\frac{h v}{2} \frac{1}{1-\exp (-h v \mid k T)}\right\} d v
\end{aligned}
$$

In working out Equation (3) we have used the assumptions,

$$
\begin{aligned}
& \mathrm{B}_{\mathrm{nm}}=\mathrm{B}_{\mathrm{mn}}=\mathrm{B}, \\
& \mathrm{A}_{\mathrm{mn}}=\mathrm{A} \text { and } \mathrm{A}_{\mathrm{nm}}=\frac{1}{2} \quad \mathrm{~A}_{\mathrm{mn}}=\frac{1}{2} \mathrm{~A}
\end{aligned}
$$

It is now worthwhile to compare Equation (3) with Equation (1). We observe that the Equation (3) is identical to Equation (1) which was derived by Plank in 1911.It contains the black body radiation formula and a second term that pointed directly to the existence of zero point energy. In equation (3) also we observe that if the temperature $\mathrm{T}$ drops to zero, we are left with the zero point energy term. Thus we find that the important consequence of using spontaneous absorption in Einstein's rate equation approximation is the zero-point energy term.

\section{CONCLUSION}

From what has been discussed above it is appropriate to draw conclusions. We have observed that in the steady state temperature $\mathrm{T}$, inclusion of spontaneous absorption term (with some arbitrarily chosen transition probability) results in the same distribution law as derived by Einstein plus an additional term which points directly to the universal phenomenon of zero-point energy. Our result is based on the bold assumption that spontaneous absorption probability is finite and nonzero. Thus it is appropriate to conclude that spontaneous absorption has some significant in connection with Einstein's Rate equation approximation. Since spontaneous absorption leads to zero point energy, under some circumstances we may state that the physics of spontaneous absorption is not yet completely understood.

\section{REFERENCES}

[1]. A. Einstein Quantum theory, Z Phys 18 (1917) 121.

[2]. V.F. Weisskopf and E Wigner, Z Phys 39, 54(1930).

[3]. W. Heitler, Quantum Theory of Radiation Oxford (1936).

[4]. V.N. Ginzburg, Soviet. Phys. Usp 26 (8) 713(1983).

[5]. W.E. Lamb Jr. and R.C Retherford, Phys Rev. 71, 241(1947).

[6]. H B G Casimir, Physica 19, 846(1953).

[7]. M. Sargent III, M.O. Scully and W.E.Lamb Jr. Laser Physics (Addison Wesley Publishing Company, Reading, Massachusetts, 1974). 
[8]. M. Planck, Verhandlungen der Deutschen Physikalischen Geselischaft, 13, 138(1911).

[9]. A Einstein and O stern, Ann. Physik 40, 551 (1913).

[10]. P.W. Miloni, Am J, Phys. 52(4) 340,(1980).

[11]. V.F. Weisskopf Natur wiss enschaften 23, 631(1935).

[12]. T.A. Welton, Phys Rev. 74, 1157(1948).

[13]. V.M. Fain and Ya I Khanin, Quantum Electronics (Mit, Cambridge, MA) Vol I (1969) Sec. 28 and 29.

[14]. VLGinzburg, Terotiches Kaya Fizika, (Theoretical Physics and Astrophysics) Nauka, Moscow(1981). 